\title{
ON A COMPUTATIONAL ALGORITHM FOR TIME-LAG OPTIMAL CONTROL PROBLEMS WITH RESTRICTED PHASE COORIDINATES
}

\author{
K. L. TEO and B. D. CRAVEN
}

(Received 26 January 1979)

(Revised 7 June 1979)

Abstract

In this paper we present a computational method for solving a class of time-lag optimal control problems with restricted phase coordinates.

\section{Introduction}

In this paper we present a computational algorithm for solving a class of time-lag optimal control problems with restricted phase coordinates. Such problems arise in the optimal control of inventory or storage systems, where the inventory is constrained to be non-negative and of ten the storage space is restricted, and also in the optimal control of a river system [3]. Another application [4] relates to the optimal control of a nuclear reactor.

The algorithm is an extension of the algorithm given in [6] for solving the same class of problem, but without the restricted phase coordinates. The idea is motivated by the projected gradient method found in classical optimization techniques. Consider a trial control function, for which the state satisfies its restrictions. If the corresponding Hamiltonian is not maximal, then the control is modified, on a suitably small time interval, so as to reduce the objective function. This leads to an iterative algorithm.

In Section 5 a very simple example is given, and solved analytically, to illustrate the method. However, numerical experience and an estimate of convergence rate are not yet available.

The present algorithm may be contrasted with that of [5], where there is a convergence result, but no estimate of convergence rate; however, [5] does not include time-delayed arguments and does not handle phase constraints. The numerical 
experience in [6] suggests that the present algorithm, which extends that of [6], should be numerically effective.

\section{Statement of the problem, basic definitions and assumptions}

Consider the following delay-differential equation on the fixed time interval $(0, T]$

$$
\dot{x}(t)=\sum_{j=0}^{s} f^{j}\left(t-h_{j}, x\left(t-h_{j}\right), u\left(t-h_{j}\right)\right)
$$

where $x \triangleq\left(x_{1}, \ldots, x_{n}\right) \in R^{n}$ and $u \Delta\left(u_{1}, \ldots, u_{r}\right) \in R^{r}$ are, respectively, the state and control vectors, and $f^{j} \Delta\left(f_{1}^{j}, \ldots, f_{n}^{j}\right) \in R^{n}, j=0,1, \ldots, s$. The $h_{k}$ are the time delays, ordered so that

$$
0=h_{0}<h_{1}<\ldots<h_{s}<T, \quad s<\infty \text {. }
$$

The initial function for the differential equation (1) is

$$
x(t)=\phi(t) \text { for } t \in\left[-h_{s}, 0\right]
$$

where $\phi \Delta\left(\phi_{1}, \ldots, \phi_{n}\right)$ is a given, absolutely continuous function on $\left[-h_{s}, 0\right]$ with values in $R^{n}$.

Let $D$ be the class of all controls defined by

$D \triangleq\left\{u: u\right.$ is a function from $\left[-h_{s}, T\right]$ into $U$, piecewise continuous on $[0, T]$ and with $u(t)=\beta(t)$ on $\left.\left[-h_{s}, 0\right)\right\}$,

where $\beta$ is a given piecewise continuous function on $\left[-h_{s}, 0\right)$ with values in $U$, and $U$ is a compact and convex subset of $R^{r}$.

By virtue of ([6], Theorem 2.3, page 318), we observe that, for each $u \in D$, system (1)-(2) admits a unique solution $x(u)($.$) . Let \Gamma \Delta\left(\Gamma_{1}, \ldots \Gamma_{p}\right)$, be a function from $[0, T] \times R^{n(s+1)}$ into $R^{p}$, where $R^{n(s+1)}=R^{n} \times \ldots \times R^{n}((s+1)$ times), and let $\mathscr{D} \subset D$ be the class of admissible controls defined by

$$
\mathscr{D}=\left\{u \in D: \Gamma_{k}(t, X(u)(t)) \leqslant 0, k=1, \ldots, p\right\},
$$

where $X(u)(t)$ denotes $\left(x(u)(t), x(u)\left(t-h_{1}\right), \ldots, x(u)\left(t-h_{p}\right)\right)$. The conditions $\Gamma_{k}(t, X(u)(t)) \leqslant 0, k=1,2, \ldots, p$, describe the state constraints.

We may now state our problem, denoted by $P$, as: Subject to the dynamic constraint (1) with the initial condition (2), find an admissible control $u \in \mathscr{D}$ that will minimize the cost functional $J$ defined by

$$
J(u)=\int_{0}^{T} \sum_{j=0}^{s} f_{0}^{j}\left(t-h_{j}, x(u)\left(t-h_{j}\right), u\left(t-h_{j}\right)\right) d t,
$$

where, for each $j \in\{0,1, \ldots, s\}, f_{0}^{j}$ is a real-valued function. 
For any $z \in R^{n},\|z\|$ denotes the Euclidean norm $\left(\sum_{i=1}^{n} z_{i}^{2}\right)^{\frac{1}{2}}$. For any function $g: R^{m} \rightarrow R^{n}, \partial g / \partial x$ denotes the Jacobian matrix whose $i, j$ element is $\partial g_{i} / \partial x_{j}$.

The following assumptions $(A)$ will be assumed throughout. The functions $f_{0}^{j}, f^{j}$ and $\partial f^{j} / \partial x, j=1, \ldots, s$, are piecewise continuous on $[0, T]$ for all $(x, u) \in R^{n} \times U$ and continuous on $R^{n} \times U$ for each $t \in[0, T]$. Moreover, for each compact set $\Omega \subset R^{n}$, there exists a positive constant $m$ so that

$$
\left\|f_{0}^{j}(t, x, v)\right\| \leqslant m(1+\|x\|) \text { and }\left\|f^{j}(t, x, v)\right\| \leqslant m(1+\|x\|) .
$$

for all $j \in\{0,1, \ldots, s\}$ and for all $(t, x, v) \in[0, T] \times \Omega \times U$. In addition, it is assumed that $\Gamma\left(., x, y^{1}, \ldots, y^{s}\right)$ is continuous on $[0, T]$ for each $\left(x, y^{1}, \ldots, y^{s}\right) \in R^{s+1}$ and $\Gamma(t, \ldots, \ldots,$.$) is twice continuously differentiable on R^{s+1}$ for each $t \in[0, T]$.

In the results to follow, we need

DeFINITION 2.1. For each $u \in D$, the absolutely continuous function

$$
\psi(u) \Delta\left(\psi_{0}(u), \psi_{1}(u), \ldots, \psi_{n}(u)\right):[0, T] \rightarrow R^{n+1}
$$

is the solution of

$$
\left.\begin{array}{l}
\dot{\psi}_{i}(t)=-\sum_{k=0}^{n} \sum_{j=0}^{s} \frac{\partial f_{k}^{j}(t, x(t), u(t))}{\partial x_{i}} \psi_{k}\left(t+h_{j}\right), \quad i=1, \ldots, n, \\
\psi_{0}(t)=-1,
\end{array}\right\}
$$

with the final condition

and

$$
\left.\begin{array}{l}
\psi_{l}(t)=0 \quad \text { for all } t \geqslant T, \quad i=1, \ldots, n \\
\psi_{0}(t)=0 \text { for all } t \geqslant T .
\end{array}\right\}
$$

DEFINITION 2.2. Using the abbreviation $\Psi(t)$ for $\left(\psi\left(t+h_{0}\right), \psi\left(t+h_{1}\right), \ldots, \psi\left(t+h_{s}\right)\right)$ we define the Hamiltonian $H:[0, T] \times R^{n} \times U \times R^{(n+1)(s+1)} \rightarrow R^{1}$ to be

$$
H(t, x, v, \Psi) \Delta \sum_{k=0}^{n} \sum_{j=0}^{s} f_{k}^{j}(t, x, v) \psi_{k}\left(t+h_{j}\right)
$$

\section{Preliminaries}

In order to devise a computational algorithm for approximating problem $P$, we need certain preparatory results to be presented in this section. For these, we need

Definition 3.1. A control $u^{*} \in \mathscr{D}$ is said to be an extremal control if it satisfies 
the following condition

$$
\begin{aligned}
\int_{0}^{T} H\left(t, x\left(u^{*}\right)(t), u^{*}(t), \Psi\left(u^{*}\right)(t)\right) d t & \\
& =\max \left\{\int_{0}^{T} H\left(t, x\left(u^{*}\right)(t), u(t), \Psi\left(u^{*}\right)(t)\right) d t: u \in \mathscr{D}\right\} .
\end{aligned}
$$

REMARK 3.2. For a $u \in \mathscr{D}$, let $\Theta^{\prime}(u)$ denote the points of discontinuity of the functions

$$
f_{i}^{j}(., x(u)(.), u(.)) \text { and } \partial f_{i}^{j}(., x(u)(.), u(.)) / \partial x, \quad i=0,1, \ldots, n, \quad j=0,1, \ldots, s .
$$

Now let $\Theta(u)$ consist of all the $\theta^{\prime} \in \Theta^{\prime}(u)$ and the points $\theta^{\prime}+h_{j}, j=1, \ldots, s, 0$ and $T$. Note that if all the functions mentioned in this remark are continuous then the set $\Theta(u)$ reduces to $\{0, T\}$.

ReMARK 3.3. When a piecewise continuous function $\gamma$, having discontinuities at $a$ and $b$, is considered in $[a, b], \gamma(a)$ shall mean $\gamma(a+0)$, and $\gamma(b)$ shall mean $\gamma(b-0)$.

In the following Remark, we divide the interval $[0, T]$ into intervals of length, at most, $h_{1}$. Then, the differential equation (1) can be treated as a differential equation without delays since all the delayed terms will occur in previous intervals and therefore will have already been calculated.

REMARK 3.4. For $u^{1} \in \mathscr{D}$, define

$$
\Phi\left(u^{1}\right)=\left\{t \in[0, T]: \Gamma_{k}\left(t, X\left(u^{1}\right)(t)\right)=0 \text { for some } k=1, \ldots, p\right\} .
$$

Let $t_{c} \in[0, T]$ be such that

(i) $t_{C}=T$ if $\max \Phi\left(u^{1}\right)=T$;

(ii) $t_{C}>\max \Phi\left(u^{1}\right)$ if $\Phi\left(u^{1}\right)$ is non-empty and $\max \Phi\left(u^{1}\right)<T$;

(iii) $t_{c} \geqslant 0$ if $\Phi\left(u^{1}\right)$ is empty.

If $\max \Phi\left(u^{1}\right)=T$, set $l_{1}=l_{2}=0$; if $\max \Phi\left(u^{1}\right)<T$, set $l_{1}=0, l_{2}>0$; if $t_{C}=0$ or if $t_{c} \in \Phi\left(u^{1}\right)$, set $l_{1}=0, l_{2}>0$. In all other cases, choose constants $l_{1}>0, l_{2}>0$. Let $\varepsilon>0$ be such that no $\theta \in \Theta\left(u^{1}\right)$ (defined in Remark 3.2) is contained in the interval $\left[t_{C}-\varepsilon l_{1}, t_{C}+\varepsilon l_{2}\right]$ as an interior point, and no intervals

$$
\left[t_{c}-\varepsilon l_{1}+h_{j}, t_{c}+\varepsilon l_{2}+h_{j}\right], j=0,1, \ldots, s,
$$

overlap. Let $w$ be a vector in $U$ such that $w \neq u^{1}\left(t_{c}\right)$. Let $\Omega_{\varepsilon}=\left[t_{c}-\varepsilon l_{1}, t_{c}+\varepsilon l_{2}\right]$ and let

$$
u_{\varepsilon}^{1}(t)= \begin{cases}w & \text { if } t \in \Omega_{\varepsilon} \\ u^{1}(t) & \text { otherwise }\end{cases}
$$


By the definition of $u_{\varepsilon}^{1}$, we observe that $u_{\varepsilon}^{1}(t)=u^{1}(t)$ on $\left[-h_{s}, t_{c}-\varepsilon l_{1}\right)$. Thus, $\Gamma\left(t, X\left(u_{\varepsilon}^{1}\right)(t)\right)=\Gamma\left(t, X\left(u^{1}\right)(t)\right)$ on $\left[0, t_{c}-\varepsilon l_{1}\right]$.

Consider the differential equation (1) on $\left[t_{c}-\varepsilon l_{1}, t_{c}+\varepsilon l_{2}\right]$. By its construction, this interval does not contain $\theta \in \Theta\left(u^{1}\right)$ as interior points and hence the righthand side of (1) is continuous for $u=u^{1}$. It is also continuous in this interval for $u=u_{\varepsilon}^{1}$ because $u_{\varepsilon}^{1}(t)=w$ on $\left[t_{c}-\varepsilon l_{1}, t_{c}+\varepsilon l_{2}\right]$. Therefore, it can be shown easily that, for all $t \in\left(t_{c}-\varepsilon l_{1}, t_{c}+\varepsilon l_{2}\right]$,

$$
x\left(u_{\varepsilon}^{1}\right)(t)=x\left(u^{1}\right)(t)+\varepsilon \xi\left(u^{1} ; w\right)\left(t_{c}\right)+o(\varepsilon)
$$

where

$$
\xi\left(u^{1} ; w\right)\left(t_{c}\right)=\left(l_{1}+l_{2}\right)\left[f^{0}\left(t_{c}, x\left(u^{1}\right)\left(t_{c}\right), w\right)-f^{0}\left(t_{c}, x\left(u^{1}\right)\left(t_{c}\right), u^{1}\left(t_{c}\right)\right)\right]+o(\varepsilon),
$$
and $o(\varepsilon)$ is such that

Define

$$
\lim _{\varepsilon \downarrow_{0}} \frac{\|o(\varepsilon)\|}{\varepsilon}=0
$$

$$
\Xi\left(u^{1} ; w\right)\left(t_{c}\right)=\left(\xi\left(u^{1} ; w\right)\left(t_{c}\right), \xi\left(u^{1} ; w\right)\left(t_{c}-h_{1}\right), \ldots, \xi\left(u^{1} ; w\right)\left(t_{c}-h_{s}\right)\right) .
$$

Since

$$
u_{\varepsilon}^{1}(t)=u^{1}(t) \text { on } \quad\left[-h_{s}, t_{c}-l_{1}\right) \text {, }
$$

it follows that

$$
x\left(u_{\varepsilon}^{1}\right)(t)=x\left(u^{1}\right)(t) \text { on }\left[-h_{s}, t_{c}-l_{1}\right] .
$$

Further, we recall that no $\left[t_{c}-\varepsilon l_{1}+h_{j}, t_{c}+\varepsilon l_{2}+h_{j}\right], j=0,1, \ldots, s$, overlap. Thus, we obtain readily that

$$
\xi\left(u^{1} ; w\right)\left(t_{c}-h_{j}\right)=0, j=1, \ldots, s .
$$

Now, by virtue of the conditions imposed on $\Gamma$ given in assumption $(A)$ and equations (8), (9), (10) and (11), we deduce from Taylor's theorem that

$$
\left.\begin{array}{rl}
\Gamma_{k}\left(t, X\left(u_{\varepsilon}^{1}\right)(t)\right) & =\Gamma_{k}\left(t, X\left(u^{1}\right)(t)+\varepsilon \Xi\left(u^{1} ; w\right)\left(t_{c}\right)+o(\varepsilon)\right) \\
= & \Gamma_{k}\left(t, X\left(u^{1}\right)(t)\right)+\varepsilon\left(\partial \Gamma_{k}\left(t, X\left(u^{1}\right)(t)\right) / \partial x\right) \\
& \times \xi\left(u^{1} ; w\right)\left(t_{c}\right)+o(\varepsilon),
\end{array}\right\}
$$

for $k=1, \ldots, p$, on $\left[t_{c}-\varepsilon l_{1}, t_{c}+\varepsilon l_{2}\right]$.

From the definition of $t_{c}$, we observe that, for all $t \in\left[t_{c}-\varepsilon l_{1}, t_{c}+\varepsilon l_{2}\right]$,

$$
\Gamma_{k}\left(t, X\left(u^{1}\right)(t)\right)<0, \quad k=1, \ldots, p .
$$

Thus, it follows from (12), (13) and assumptions $(A)$ that there exists an $\varepsilon_{1}>0$ so that

$$
\Gamma_{k}\left(t, X\left(u_{\varepsilon}^{1}\right)(t)\right) \leqslant 0, \quad k=1, \ldots, p,
$$

on $\left[t_{c}-\varepsilon l_{1}, t_{c}+\varepsilon l_{2}\right]$ for all $\varepsilon, 0 \leqslant \varepsilon<\varepsilon_{1}$. 
By the definition of $u_{\varepsilon}^{1}$ given in (7), we observe that

$$
u_{e}^{1}(t)=u^{1}(t) \text { for all } t \in\left(t_{c}+\varepsilon l_{2}, t_{c}-\varepsilon l_{1}+h_{1}\right] .
$$

Therefore, the difference between the trajectories $x\left(u^{1}\right)($.$) and x\left(u_{\varepsilon}^{1}\right)($.$) on this$ interval will result only from the differing initial conditions for the differential equation (1) on $\left(t_{c}+\varepsilon l_{2}, t_{c}-\varepsilon l_{1}+h_{1}\right]$. The perturbation of the initial conditions is given in equation (8) with $t=t_{c}+\varepsilon l_{2}$. Thus, we may use ([1], Lemma 4.3 with $r=1, \delta t_{0}=\delta t_{1}=0, \delta F(t, y)=0$ and $g(t, y, \alpha, \varepsilon)=0$, pages 258-259) to obtain

$$
x\left(u_{\varepsilon}^{1}\right)(t)=x\left(u^{1}\right)(t)+\varepsilon \delta x_{i}\left(u^{1}\right)(t)+o(\varepsilon)
$$

for $t \in\left(t_{c}+\varepsilon l_{2}, t_{c}-\varepsilon l_{1}+h_{1}\right]$, where

$$
\delta \dot{x}\left(u^{1} ; w\right)(t)=\sum_{k=1}^{n} \sum_{j=0}^{s} \frac{\partial f^{j}\left(t-h_{j}, x\left(u^{1}\right)\left(t-h_{j}\right), u^{1}\left(t-h_{j}\right)\right)}{\partial x_{k}} \delta x_{k}\left(u^{1} ; w\right)\left(t-h_{j}\right)
$$

for $t \in\left(t_{c}+\varepsilon l_{2}, t_{c}-\varepsilon l_{1}+h_{1}\right]$, with the initial conditions

$$
\begin{aligned}
& \left.\delta x\left(u^{1} ; w\right)(t)=\xi\left(u^{1} ; w\right)\left(t_{c}\right), \quad t \in\left(t-\varepsilon l_{1}, t_{c}+\varepsilon l_{2}\right],\right\} \\
& \left.\delta x\left(u^{1} ; w\right)(t)=0, \quad t \in\left[0, t-\varepsilon l_{1}\right] .\right\}
\end{aligned}
$$

Define

$$
\delta X\left(u^{1} ; w\right)(t) \underline{\Delta}\left(\delta x\left(u^{1} ; w\right)(t), \delta x\left(u^{1} ; w\right)\left(t-h_{1}\right), \ldots, \delta x\left(u^{1} ; w\right)\left(t-h_{s}\right)\right) .
$$

Then it follows that

$$
\delta X\left(u^{1} ; w\right)(t)=\left(\delta x\left(u^{1} ; w\right)(t), 0, \ldots, 0\right) \quad \text { on }\left(t_{c}+\varepsilon l_{2}, t_{c}-\varepsilon l_{1}+h_{1}\right] .
$$

Now we note that, for all $t \in\left(t_{c}+\varepsilon l_{2}, t_{c}-\varepsilon l_{1}+h_{1}\right]$,

$$
\Gamma_{k}\left(t, X\left(u_{\varepsilon}^{1}\right)(t)\right)=\Gamma_{k}\left(t, X\left(u^{1}\right)(t)+\varepsilon \delta X\left(u^{1} ; w\right)(t)+o(\varepsilon)\right), \quad k=1, \ldots, p,
$$

where $\delta X\left(u^{1} ; w\right)(t)$ is given by (19). Next, in view of assumption $(A)$ and the definition of $\xi\left(u^{1} ; w\right)\left(t_{c}\right)$ given in (9), it follows from (16) to (17) and Gronwall's inequality that $\left\|\delta x\left(u^{1} ; w\right)(t)\right\|$ is bounded on $\left[t_{c}+\varepsilon l_{2}, t_{c}-\varepsilon l_{1}+h_{1}\right]$ uniformly with respect to $\varepsilon, 0 \leqslant \varepsilon \leqslant \varepsilon_{1}$. Thus, by virtue of the conditions imposed on $\Gamma$ given in assumptions $(A),(20)$, Taylor's theorem and the fact just mentioned above, we deduce from the definition of $t_{c}$ that there exists an $\varepsilon_{2}, 0<\varepsilon_{2} \leqslant \varepsilon_{1}$, such that

$$
\Gamma_{k}\left(t, X\left(u_{\varepsilon}^{1}\right)(t)\right) \leqslant 0, \quad k=1, \ldots, p,
$$

on $\left(t_{c}+\varepsilon l_{2}, t_{c}-\varepsilon l_{1}+h_{1}\right]$ for all $\varepsilon, 0 \leqslant \varepsilon<\varepsilon_{2}$.

This procedure is repeated for an interval of length $h_{1}$ (in this case, we consider the interval $\left.\left(t_{c}+\varepsilon l_{2}+h_{1}, t_{c}+\varepsilon l_{2}+2 h_{1}\right]\right)$, or until the point $t_{c}-\varepsilon l_{1}+h_{2}$ is reached 
(in this case, we consider the interval $\left.\left(t_{c}+\varepsilon l_{2}+h_{1}, t_{c}-\varepsilon l_{1}+h_{2}\right]\right)$, or until the final time $T$ is reached (in this case, we consider the interval $\left(t_{c}+\varepsilon l_{2}+h_{1}, T\right]$ ). The choice is made so that the interval is smallest. Then, as explained before, in this interval the delay-differential equation can be treated as an ordinary differential equation. If we reach $t_{c}-\varepsilon l_{1}+h_{2}$ before proceeding a length $h_{1}$ and before the final time $T$ is reached, we derive new versions of equations (8) and (9) on

This is necessary because in the interval

$$
\left(t_{c}-\varepsilon l_{1}+h_{2}, t_{c}+\varepsilon l_{2}+h_{2}\right] \text {. }
$$

$$
\left(t_{c}-\varepsilon l_{1}+h_{2}, t_{c}+\varepsilon l_{2}+h_{2}\right]
$$

the time delays have the effect of perturbing the differential system. On a basis of the new versions of equations (8) and (9) on $\left(t_{c}-\varepsilon l_{1}+h_{2}, t_{c}+\varepsilon l_{2}+h_{2}\right]$, it follows from a similar argument that there exists an $\varepsilon_{3}>0$ so that (14) remains valid on $\left(t_{c}-\varepsilon l_{1}+h_{2}, t_{c}+\varepsilon l_{2}+h_{2}\right]$ for all $\varepsilon, 0 \leqslant \varepsilon<\varepsilon_{3}$.

The whole process is kept on repeating until we reach the final time $T$ before (or on exactly) proceeding a length $h_{1}$ and before (or exactly as) any of the points $t_{c}-\varepsilon l_{1}+h_{j}, j=3, \ldots, s$, is reached. At this stage, the argument is complete and we can conclude that there exists an $\hat{\varepsilon}>0$ such that

$$
\Gamma_{k}\left(t, X\left(u_{\varepsilon}^{1}\right)(t)\right) \leqslant 0, \quad k=1, \ldots, p,
$$

on $[0, T]$ for all $\varepsilon, 0 \leqslant \varepsilon<\hat{\varepsilon}$. This, in turn, implies that $u_{\varepsilon}^{1} \in \mathscr{D}$ whenever $\varepsilon$ is such that $0 \leqslant \varepsilon<\hat{\varepsilon}$.

\section{Motivation for the Algorithm}

In this section we present our main result, which is Theorem 4.1. This theorem proves that, under certain conditions, if we are given a control $u^{1} \in \mathscr{D}$ that is not an extremal control, then a new control $u^{2} \in \mathscr{D}$ can be constructed so that the corresponding objective functional will be improved. On the basis of Theorem 4.1, we present a computational algorithm for approximating a solution of problem $P$ in Section 5.

THEOREM 4.1. Consider problem $P$. Suppose that assumptions $(A)$ are satisfied and that $u^{\mathbf{1}} \in \mathscr{D}$ is not an extremal control. Let $\omega$ be a vector in $U$ such that $\omega \neq u^{\mathbf{1}}\left(t_{c}\right)$ and such that

$$
H\left(t_{c}, x\left(u^{1}\right)\left(t_{c}\right), \omega, \Psi\left(u^{1}\right)\left(t_{c}\right)\right)>H\left(t_{c}, x\left(u^{1}\right)\left(t_{c}\right), u^{1}\left(t_{c}\right), \Psi\left(u^{1}\right)\left(t_{c}\right)\right) .
$$

Further, let $\Omega_{\varepsilon}=\left[t_{c}-\varepsilon l_{1}, t_{c}+\varepsilon l_{2}\right]$ and let

$$
u_{\varepsilon}^{1}(t)= \begin{cases}\omega & \text { if } t \in \Omega_{\varepsilon}, \\ u^{1}(t) & \text { otherwise, }\end{cases}
$$


where $t_{c}, \varepsilon, l_{1}$ and $l_{2}$ are as defined in Remark 3.4. Then,

$$
\begin{aligned}
& J\left(u^{1}\right)-J\left(u_{s}^{1}\right) \\
& =\int_{\Omega}\left\{H\left(t, x\left(u^{1}\right)(t), \omega, \Psi\left(u^{1}\right)(t)\right)-H\left(t, x\left(u^{1}\right)(t), u^{1}(t), \Psi\left(u^{1}\right)(t)\right)\right\} d t+o(\varepsilon)
\end{aligned}
$$

Further, there exists an $\varepsilon_{0}>0$ such that

on $[0, T]$ and

$$
\Gamma_{k}\left(t, X\left(u_{\varepsilon}^{1}\right)(t)\right) \leqslant 0, \quad k=1, \ldots, p,
$$

$$
J\left(u^{1}\right)>J\left(u_{\varepsilon}^{1}\right)
$$

for all $\varepsilon, 0<\varepsilon<\varepsilon_{0}$.

Proof. The proof for the first part of the theorem is similar to that of Theorem 4.5 of [2] with only some obvious modifications.

In view of Remark 3.4, we observe that there exists an $\hat{\varepsilon}>0$ such that (25) holds true on $[0, T]$ for all $\varepsilon, 0 \leqslant \varepsilon<\hat{\varepsilon}$.

Next, we note that the functions

$$
H\left(., x\left(u^{1}\right)(.), \omega, \Psi\left(u^{1}\right)(.)\right) \text { and } H\left(., x\left(u^{1}\right)(.), u^{1}(.), \Psi\left(u^{1}\right)(.)\right)
$$

are continuous on $\left(t_{c}-\varepsilon l_{1}, t_{c}+\varepsilon l_{2}\right)$. Thus, by virtue of (24) and (23), it follows that there exists an $\hat{\varepsilon}>0$ such that

for all $\varepsilon, 0<\varepsilon<\hat{\hat{\varepsilon}}$.

$$
\frac{1}{\varepsilon\left(l_{1}+l_{2}\right)}\left[J\left(u^{1}\right)-J\left(u_{\varepsilon}^{1}\right)\right]>0
$$

Thus, the proof is complete by choosing $\varepsilon_{0}=\min \{\hat{\varepsilon}, \hat{\varepsilon}\}$.

By repeated application of Theorem 4.1, we can construct from any admissible control $u^{0} \in \mathscr{D}$ a sequence of admissible controls $\left\{u^{k}\right\} \subset \mathscr{D}$ such that

$$
J\left(u^{k}\right)>J\left(u^{k+1}\right) \text { for all } k=1,2, \ldots
$$

REMARK 4.2. In Theorem 4.1, we do not require that $\omega$ is a vector that maximizes $H\left(t_{c}, x\left(u^{1}\right)\left(t_{c}\right), ., \Psi\left(u^{1}\right)\left(t_{c}\right)\right)$ globally on $U$. Thus, the maximization of $H\left(t, x\left(u^{1}\right)(t), ., \Psi\left(u^{1}\right)(t)\right)$ with respect to $v \in U$ at each net point constructed in step 7 of the algorithm below can be performed by any practical method, for example, the projected gradient method, when the calculus methods fail to be accessible in practice.

\section{The algorithm}

On the basis of Theorem 4.1, we present a computational algorithm for approxi- 
mating a solution of problem $P$ as follows:

1. Select an $\varepsilon>0$.

2. Choose a $u^{0} \in D$ such that

$$
\Gamma_{k}\left(t, X\left(u^{0}\right)(t)\right) \leqslant 0, \quad k=1, \ldots, p,
$$

for all $t \in[0, T]$, where $X\left(u^{0}\right)(t)=\left(x\left(u^{0}\right)(t), x\left(u^{0}\right)\left(t-h_{1}\right), \ldots, x\left(u^{0}\right)\left(t-h_{s}\right)\right)$ and $x\left(u^{0}\right)($.$) is the solution of system (1)-(2) corresponding to u^{0}$. Set

$$
t_{0}^{*}=\max \left\{\tau: \tau \in \Phi\left(u^{0}\right)\right\}
$$

if $\Phi\left(u^{0}\right)$ is non-empty and $t_{0}^{*}=0$ if $\Phi\left(u^{0}\right)$ is empty. If $t_{0}^{*}=T$, go to step 18. Otherwise go to step 3 .

3. Set $\alpha=0$.

4. Calculate $\psi\left(u^{\alpha}\right)($.$) by solving the system (4)-(5) corresponding to u^{\alpha}$ backward in time.

5. Calculate

$$
\beta_{\gamma}=\left[\frac{\hat{\theta}_{\gamma+1}-\hat{\theta}_{\gamma}}{\varepsilon}\right]+1
$$

for each $\hat{\theta}_{\gamma} \in \widehat{\Theta}\left(u^{\alpha}\right)$, where [a] means the greatest integer less than or equal to $a$ and $\Theta\left(u^{\alpha}\right)$ consists of all the points in $\Theta\left(u^{\alpha}\right)$ and $t_{\alpha}^{*}$. Also find $B=\sum_{\gamma=0}^{\gamma^{\prime}} \beta_{\gamma}$, where $\gamma^{\prime}$ is the largest value that $\gamma$ takes.

6. Compute $\Delta_{\gamma}$ by

7. Define

$$
\Delta_{\gamma}=\frac{\hat{\theta}_{\gamma+1}-\hat{\theta}_{\gamma}}{\beta_{\gamma}}, \quad \gamma=0,1, \ldots, \gamma^{\prime} .
$$

$$
b\left(\gamma, \zeta(\gamma)=\hat{\theta}_{y}+\zeta(\gamma) \Delta_{\gamma}\right.
$$

where $\gamma=0,1, \ldots, \gamma^{\prime}$ and $\zeta(\gamma)=0,1, \ldots, \beta_{\gamma}-1$. Now assign

$$
b(1)=\hat{b}(0,0), b(2)=\hat{b}(0,1), \ldots, b(B)=\hat{b}\left(\gamma^{\prime}, \beta_{\gamma},-1\right) .
$$

These are denoted as $b(m), m=1, \ldots, B$.

8. Find $m_{1} \in\{1, \ldots, B\}$ such that $b\left(m_{1}\right)=t_{\alpha}^{*}$.

9. Compute the $\omega\left(u^{\alpha}\right)(b(m)), m=m_{1}, \ldots, B$, by any practical method, where $\omega\left(u^{a}\right)(b(m))$ is a solution of

$$
\max \left\{H\left(b(m), x\left(u^{\alpha}\right)(b(m)), v, \psi\left(u^{\alpha}\right)(b(m))\right): v \in U\right\} .
$$

10. (i) If $b\left(m_{1}\right)=0$, find the first $m_{2} \in\left\{m_{1}, \ldots, B\right\}$ such that $\Delta H\left(b\left(m_{2}\right)\right)>0$, where

$$
\begin{aligned}
\Delta H(b(m))=H\left(b(m), x\left(u^{\alpha}\right)(b(m)),\right. & \left.\omega\left(u^{\alpha}\right)(b(m)), \psi\left(u^{\alpha}\right)(b(m))\right) \\
& -H\left(b(m), x\left(u^{\alpha}\right)(b(m)), u^{\alpha}(b(m)), \psi\left(u^{\alpha}\right)(b(m))\right) .
\end{aligned}
$$

(ii) If $b\left(m_{1}\right)>0$, find the first $m_{2} \in\left\{m_{1}+1, \ldots, B\right\}$ such that $\Delta H\left(b\left(m_{2}\right)\right)>0$. 
11. Set $\kappa=0$ and define

$$
u^{\alpha, 0}(t)= \begin{cases}\omega\left(u^{\alpha}\right)(b(m)), & t \in[b(m), \dot{b}(m+1)], \\ \boldsymbol{u}^{\alpha}(t), & \text { elsewhere. }\end{cases}
$$

Go to step 13.

12. Define

$$
u^{\alpha, \kappa}(t)= \begin{cases}\omega\left(u^{\alpha}\right)(b(m)), & t \in[b(m), b(m+1)) \\ u^{\alpha}(t), & \text { for } m \in\left\{m_{2}, \ldots,\left[\frac{B}{2^{\kappa}}\right]\right\}\end{cases}
$$

13. If $u^{\alpha, x}$ is such that

$$
\Gamma_{k}\left(t, X\left(u^{\alpha, \kappa}\right)(t)\right) \leqslant 0, \quad k=1, \ldots, p,
$$

for all $t \in[0, T]$, where $\left.X\left(u^{\alpha, \kappa}\right)(t)\right)=\left(x\left(u^{\alpha, \kappa}\right)(t), x\left(u^{\alpha, \kappa}\right)\left(t-h_{1}\right), \ldots, x\left(u^{\alpha, \kappa}\right)\left(t-h_{s}\right)\right)$ and $x\left(u^{\alpha, \kappa}\right)($.$) is the solution of the system (1)-(2) corresponding to u^{\alpha, \kappa}$, set

$$
t_{\alpha, \kappa}^{*}= \begin{cases}\max \left\{\tau: \tau \in \Phi\left(u^{\alpha, \kappa}\right)\right\} & \text { if } \Phi\left(u^{\alpha, \kappa}\right) \text { is non-empty } \\ 0 & \text { if } \Phi\left(u^{\alpha, \kappa}\right) \text { is empty, }\end{cases}
$$

and go to step 15. Otherwise go to step 14.

14. Set $\kappa=\kappa+1$ and go to step 12 .

15. (i) If $t_{\alpha, \kappa}^{*}=T$ and $J\left(u^{\alpha, \kappa}\right)<J\left(u^{\alpha}\right)$, set $u^{\alpha+1}=u^{\alpha, \kappa}$ and go to step 18 .

(ii) If $t_{\alpha, \kappa}^{*}=T$ and $J\left(u^{\alpha, \kappa}\right) \geqslant J\left(u^{\alpha}\right)$, set $u^{\alpha+1}=u^{\alpha}$ and go to step 18 .

(iii) If $t_{\alpha, \kappa}^{*}<T$, go to step 16 .

16. If $J\left(u^{\alpha, \kappa}\right)<J\left(u^{\alpha}\right)$, set $u^{\alpha+1}=u^{\alpha, \kappa}, t_{\alpha+1}^{*}=t_{\alpha, \kappa}^{*}$ and go to step 3. Otherwise go to step 17.

17. If $\left[B / 2^{\kappa+1}\right]=0$, set $\varepsilon=\varepsilon / 2$ and go to step 5. Otherwise set $\kappa=\kappa+1$ and go to step 12 .

18. Stop.

REMARK 5.1. In this algorithm, we prefer to choose an initial admissible control $u^{0}$ such that the set $\Phi\left(u^{0}\right)$ is empty. Since the improvement of the objective functional is achieved by perturbing the control $u^{0}$, it appears that the closer $t_{0}^{*}$ to $T$ the smaller the room will be left for improving the objective functional. Thus, in general, the optimal admissible control obtained from an initial admissible control $u_{0}$ such that $\Phi\left(u^{0}\right)$ is empty will be superior to that obtained from an initial admissible control $u_{0}$ such that $t_{0}^{*}$ is very close to $T$.

At this stage, it should be noted that we do not have a general method for finding an initial admissible control $u^{0}$ such that $\Phi\left(u^{0}\right)$ is empty. However, the finding of such an initial admissible control is possible for many practical problems. 
REMARK 5.2. In steps 2,4 and 13 , when we calculate $x(u)($.$) and \psi(u)($.$) , we$ first break up the interval $[0, T]$ into $\left[\theta_{j}, \theta_{j+1}\right]$ for all $\theta_{j}, \theta_{j+1} \in \Theta(u)$, where $\Theta(u)$ is as defined in Remark 3.2. Then, the system (1)-(2) corresponding $u$ is solved forward in time whereas the system (4)-(5) corresponding to $u$ is solved backward in time, both integrations being done over the previous subintervals in appropriate orders.

REMARK 5.3. With the state and costate variables known, an approximate maximizing control $\omega\left(u^{\alpha}\right)$ is calculated at each net point by any practical method. These net points are constructed in steps 5-7. In view of step 5, we note that the construction of these net points depends on the quantity $\varepsilon$.

REMARK 5.4. On the basis of Theorem 4.1, we observe that, if $u^{\alpha} \in \mathscr{D}$ and $t_{\alpha}^{*}<T$. then the algorithm will produce an improved control $u^{\alpha+1} \in \mathscr{D}$ after only a finite number of iterations. However, if $t_{a}^{*}=T$, then no improvement can be made in the value of the objective functional and hence the algorithm will terminate.

REMARK 5.5. In practice, the algorithm will terminate if $t_{\alpha, k}^{*}=T$ or $t_{\alpha}^{*}=T$ or any of the following conditions is satisfied:

(i) The improvement in objective functional per iteration is less than some tolerance.

(ii) The value of $\Delta_{\gamma}$ is less than or equal to the step size of integration.

(iii) The value of $\int_{0}^{T} \Delta H(t) d t$ is less than some tolerance.

RemarK 5.6. Note that the Hamiltonian function used in this algorithm is the one for problems without restricted phase coordinates. Thus it will be more interesting and more natural if the generalized Hamiltonian function of the typc developed in Chapter 5 of [3] can be used to derive a computational method even for the case without time delayed arguments. This remains an open problem.

\section{An example}

To check the algorithm presented in Section 5, let us consider the following simple example.

$$
\begin{aligned}
& \underset{u \in D}{\operatorname{Minimize}} J[u]=\int_{0}^{1}\{-x(u)(t)\} d t \\
& \text { subject to }\left\{\begin{array}{l}
\dot{x}(u)(t)=u(t), \quad 0 \leqslant t \leqslant 1, \\
x(u)(0)=0, \\
x(u)(t) \leqslant \frac{3}{4}, \quad 0 \leqslant t \leqslant 1,
\end{array}\right.
\end{aligned}
$$


where $u \in D$ and $D$ is the class of controls defined by

$$
\begin{aligned}
& D=\{u: u \text { piecewise continuous real-valued function defined on }[0,1] \text { and } \\
& \qquad u(t) \in[0,1] \text { for all } t \in[0,1]\} .
\end{aligned}
$$

The Hamiltonian for this example is

$$
H(t, x, u, \psi)=x+u \psi \text {. }
$$

The adjoint system is

$$
\left.\begin{array}{l}
\dot{\psi}(u)(t)=1, \quad t \in[0,1], \\
\psi(u)(1)=0 .
\end{array}\right\}
$$

Since (6.6) is independent of $u$, it can be written as

$$
\left.\begin{array}{l}
\dot{\psi}(t)=-1, \quad t \in[0,1], \\
\psi(1)=0 .
\end{array}\right\}
$$

The solution of $(6.7)$ is

$$
\psi(t)=1-t
$$

Now let us solve this example step by step according to the algorithm presented in Section 5.

From step 1: Choose $\varepsilon=2$.

From step 2: Choose

$$
u^{0}(t)= \begin{cases}0, & t \in\left[0, \frac{1}{8}\right) \\ 1, & t \in\left[\frac{1}{8}, \frac{3}{4}\right], \\ 0, & t \in\left[\frac{3}{4}, 1\right]\end{cases}
$$

Clearly, $u^{0} \in \mathscr{D}$ and $t_{0}^{*}=0$.

From step 4: $\psi(t)=1-t$.

From steps 5-7: $b(1)=0, b(2)=\frac{1}{8}$ and $b(3)=\frac{3}{4}$.

From step 8: $m_{1}=1$ and $b(1)=0=t_{v}^{*}$.

From step 9: $\omega\left(u^{0}\right)(0)=1, \omega\left(u^{0}\right)\left(\frac{1}{8}\right)=1$ and $\omega\left(u^{0}\right)\left(\frac{3}{4}\right)=1$.

From step 10: $\Delta H(0)=1>0$.

From step 11: $u^{0,0}(t)=1$ for all $t \in[0,1]$.

From step 13: $x\left(u^{0,0}\right)(t)=t$. However, $x\left(u^{0,0}\right)(t)>\frac{3}{4}$ for all $t \in\left(\frac{3}{4}, 1\right]$.

From step 12:

$$
u^{0,1}(t)= \begin{cases}1, & t \in\left[0, \frac{1}{8}\right) \\ 1, & t \in\left[\frac{1}{8}, \frac{3}{4}\right), \\ 0, & t \in\left[\frac{3}{4}, 1\right]\end{cases}
$$

From step 13:

$$
x\left(u^{0,1}\right)(t)= \begin{cases}t, & t \in\left[0, \frac{3}{4}\right), \\ \frac{3}{4}, & t \in\left[\frac{3}{4}, 1\right] .\end{cases}
$$


Clearly, $u^{0,1} \in \mathscr{D}$ and $t_{0,1}^{*}=1$.

From step 15(i): $J\left(u^{0,1}\right)=-\frac{15}{32}<J\left(u^{0}\right)=-\frac{57}{128}$.

From step 18: Stop.

In this example, the control $u^{0,1}$ obtained by the algorithm presented in Section 5 is the optimal control. However, in general, we can only expect to compute a sub-optimal control for a given control problem.

\section{References}

[1] L. D. Berkovitz, Optimal control theory (Springer-Verlag, New York, 1974).

[2] B. D. Craven, Mathematical programming and control theory (Chapman and Hall, London Mathematics Series, 1978).

[3] W. H. Farr and M. A. Hanson, "Continuous time programming with nonlinear time delayed constraints", J. Math. Anal. Appl. 46 (1974), 41-61.

[4] H. Maurer and U. Heidemann, "Optimale Steuerprozesse mit Zustandbeschränkungen", Lecture notes in mathematics 477 (Springer-Verlag, Berlin, 1975), pp. 244-259.

[5] D. Q. Mayne and E. Polak, "First-order strong variation algorithms for optimal control", J. Optim. Theor. Appl. 16 (1975), 277-301.

[6] J. M. Murray and K. L. Teo, "On a computational algorithm for a class of optimal control problems involving discrete time delayed arguments", J. Austral. Math. Soc. B 20 (1978), 315-343.

School of Mathematics

University of NSW

Kensington

NSW 2033

and

Department of Mathematics

University of Melbourne

Parkville

Victoria 3052 